This item was submitted to Loughborough's Research Repository by the author.

Items in Figshare are protected by copyright, with all rights reserved, unless otherwise indicated.

\title{
Constant modulus blind equalisation algorithms under soft constraint
} satisfaction

PLEASE CITE THE PUBLISHED VERSION

PUBLISHER

(c) IEEE

VERSION

VoR (Version of Record)

LICENCE

CC BY-NC-ND 4.0

\section{REPOSITORY RECORD}

Tanrikulu, Oguz, Buyurman Baykal, A.G. Constantinides, and Jonathon Chambers. 2019. "Constant Modulus Blind Equalisation Algorithms Under Soft Constraint Satisfaction”. figshare. https://hdl.handle.net/2134/5862. 
This item was submitted to Loughborough's Institutional Repository (https://dspace.lboro.ac.uk/) by the author and is made available under the following Creative Commons Licence conditions.

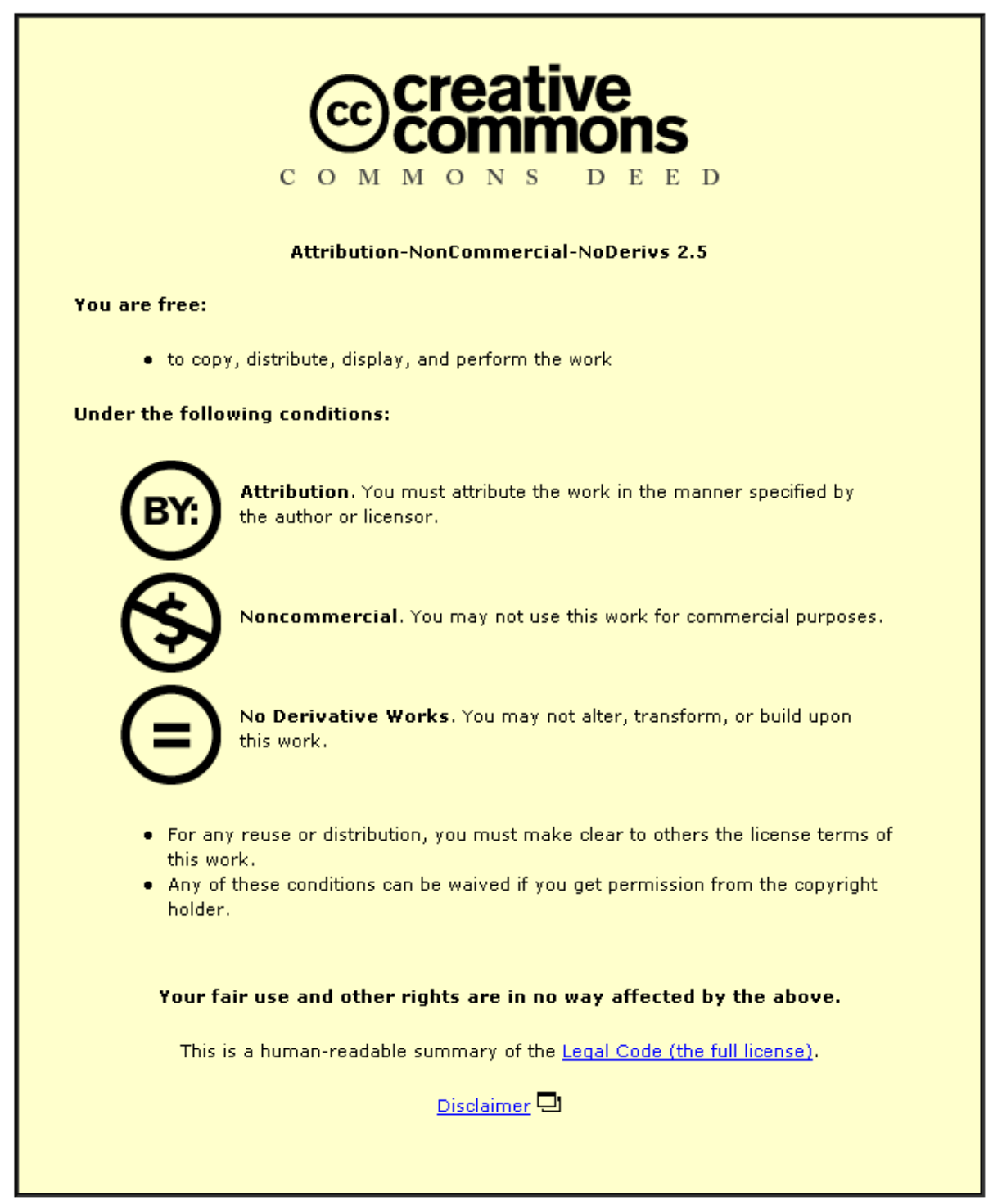

For the full text of this licence, please go to: http://creativecommons.org/licenses/by-nc-nd/2.5/ 


\title{
CONSTANT MODULUS BLIND EQUALISATION ALGORITHMS UNDER SOFT CONSTRAINT SATISFACTION
}

\author{
Oğuz Tanrıkulu, Buyurman Baykal, Anthony G. Constantinides and Jonathon A. Chambers
}

\author{
Signal Processing and Digital Systems Section, \\ Department of Electrical and Electronic Engineering, \\ Imperial College of Science, Technology and Medicine, London SW7 2BT, UK. \\ o.tanrikulu@ic.ac.uk
}

\begin{abstract}
New Constant Modulus (CM) algorithms are presented that are based on soft constraint satisfaction. The stationary points of an algorithm in this family is studied for an $\operatorname{AR}(p)$ channel and it is shown that Ding-type Undesirable Local Solutions (ULS) do not exist. This is due to the normalisation of the gradient vector and the soft nonlinearity used in these algorithms. Error Performance Surfaces (EPS) and convergence trajectories from arbitrary initialisations are presented for various channels that support the analytical findings.
\end{abstract}

\section{INTRODUCTION}

Numerous Blind Equalisation (BE) algorithms have been developed for the recovery of digitally transmitted data $[5,9,3,1,8,10]$. The BE problem is conventionally formulated within the context of the baseband model of transmission shown in Fig. 1 where $a_{k}$ is the transmitted discrete symbols, $w_{k}$ is the channel noise, $x_{k}$ is the equaliser input, $y_{k}$ is the equaliser output and $\hat{a}_{k}$ is the output of the decision device. The equalisation objective is to achieve $\hat{a}_{k}=a_{k-\Delta}$, where $\Delta$ is a delay factor.

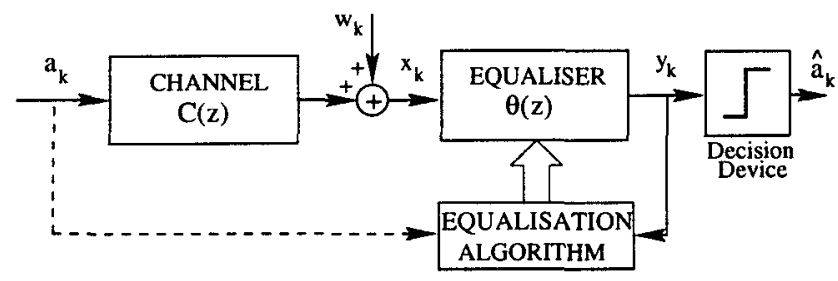

Figure 1: Baseband model for digital communications.

The existing algorithms that minimise a nonlinear function of the equaliser output $y_{k}$ possess ULS and the "eye" is often left "closed" after convergence. The Normalised CM algorithm (NCMA) proposed in $[4,7,6]$ is based on solving the deterministic optimisation problem

$$
\min _{\theta_{k+1}}\left\{\left\|\theta_{k+1}-\theta_{k}\right\|_{2}^{2}\right\} \text { st. }\left|X_{k}^{H} \theta_{k+1}\right|^{p}=1, p=1,2,3, \ldots
$$

where $\theta_{k}^{T}=\left[\begin{array}{llll}\theta_{0 \mid k} & \theta_{1 \mid k} & \ldots & \theta_{N_{\theta}-1 \mid k}\end{array}\right]$ is the equaliser vector at time instant $k$ and $X_{k}^{H}=\left[\begin{array}{llll}x_{k} & x_{k-1} & \ldots & x_{k-N_{\theta}+1}\end{array}\right]$ is the input vector to the equaliser. We use respectively $(\cdot)^{T}$ and $(\cdot)^{H}$ to denote the transpose and the Hermitian transpose of a vector.

For any $p \in \mathbb{Z}^{+}$, the a posteriori constraint $\left|X_{k}^{H} \theta_{k+1}\right|^{p}=$ 1 leads to the update equation

$$
\theta_{k+1}=\theta_{k}+\frac{\mu}{\left\|X_{k}\right\|_{2}^{2}} \underbrace{\overbrace{\operatorname{sgn}\left(y_{k}\right)}^{\psi\left(y_{k}\right)}-y_{k})}_{\Psi\left(y_{k}\right)} X_{k}
$$

where $\Psi\left(y_{k}\right)$ is the "error function" and $\psi\left(y_{k}\right)$ is the Zeromemory Non-Linearity (ZNL). In [6], (2) (with $\mu=1$ ) is shown to be an exact solution of (1). It is important to observe, however, that there is no formal justification in the formulation of the blind equalisation problem as in (1) other than around a Desirable Local Solution (DLS) where the constraint $\left|X_{k}^{H} \theta_{k+1}\right|=1$ makes sense since $\theta_{k}$ is generally varying much more slowly than $X_{k}$ and hence $\left|X_{k}^{H} \theta_{k}\right| \approx\left|X_{k}^{H} \theta_{k+1}\right|=1$. On the other hand, when $\theta_{k}$ is far from a DLS, it is not necessary to enforce $\left|X_{k}^{H} \theta_{k+1}\right|=1$ at each iteration. In NCMA, however, the a posterioni constraint determines the adaptation rule regardless of the proximity of the equaliser weights to a DLS. Although a step-size is introduced at the last-stage in [6] that softens the constraint, this does not change the properties of the ZNL and hence the EPS.

\section{SOFT CONSTRAINT SATISFACTION (SCS) ALGORITHM}

Consider the deterministic Lagrange optimisation problem

$$
\min _{\theta_{k+1}}\left\{\left\|\theta_{k+1}-\theta_{k}\right\|_{2}^{2}+\lambda\left(X_{k}^{H} \theta_{k+1} \theta_{k+1}^{H} X_{k}-R^{2}\right)\right\}
$$

where $\lambda$ is the Lagrange multiplier and $R$ is a dispersion constant that is defined in the sequel. We have

$$
y_{k} \triangleq X_{k}^{H} \theta_{k}, s_{k} \triangleq X_{k}^{H} \theta_{k+1}
$$

By differentiating (3) with respect to the real and imaginary parts of $\theta_{k+1}$, one can obtain

$$
\theta_{k+1}-\theta_{k}+\lambda X_{k} X_{k}^{H} \theta_{k+1}=0
$$


If (5) pre-multiplied by $X_{k}^{H}$, the optimum Lagrange multiplier, $\lambda_{*}$, can be solved as

$$
\lambda_{*}=-\frac{1}{\left\|X_{k}\right\|_{2}^{2}}\left(1-\frac{y_{k}}{s_{k}}\right)
$$

The corresponding update equation is

$$
\theta_{k+1}=\theta_{k}-\lambda_{*} X_{k} X_{k}^{H} \theta_{k+1}
$$

For a non-trivial update which minimises $\left\|\theta_{k+1}-\theta_{k}\right\|_{2}^{2}$ exactly, we have $s_{k}=R \operatorname{sgn}\left(y_{k}\right)$ where $\operatorname{sgn}\left(r e^{j \omega}\right) \triangleq e^{j \omega}[6]$. Thus, the ZNL is determined since (6) becomes

$$
\lambda_{*}=-\frac{1}{\left\|X_{k}\right\|_{2}^{2}}\left(1-\frac{\left|y_{k}\right|}{R}\right)
$$

Unlike NCMA, we use a soft constraint (by introducing a step-size, $\mu$, in (6), (7)) and write (7) by substituting (8) as

$$
\theta_{k+1}=\theta_{k}+\frac{\mu}{\left\|X_{k}\right\|_{2}^{2}}\left(1-\frac{\left|y_{k}\right|}{R}\right) X_{k} X_{k}^{H} \theta_{k+1}
$$

If $X_{k}^{H} \theta_{k+1}=R \operatorname{sgn}\left(y_{k}\right)$ is used on the r.h.s of (9), we get the NCMA update which is known to possess ULS. However, after introducing $\mu, X_{k}^{H} \theta_{k+1} \neq R \operatorname{sgn}\left(y_{k}\right)$ unless $\mu=1$. Therefore, we divert to another route by pre-multiplying (9) with $X_{k}^{H}$ which yields

$$
X_{k}^{H} \theta_{k+1}=\frac{y_{k}}{1-\mu\left(1-\left|y_{k}\right| / R\right)}
$$

Therefore instead of substituting $X_{k}^{H} \Theta_{k+1}=R \operatorname{sgn}\left(y_{k}\right)$ in (9), we use (10) and obtain the update equation of the SCS algorithm as

$$
\begin{gathered}
\theta_{k+1}=\theta_{k}+\frac{1}{\left\|X_{k}\right\|_{2}^{2}} \Psi\left(y_{k}\right) X_{k} \\
\Psi\left(y_{k}\right) \triangleq \frac{y_{k}}{1-\mu\left(1-\frac{\left|y_{k}\right|}{R}\right)}-y_{k}
\end{gathered}
$$

Note that, if we substitute $\mu=1$ in (11), the SCS algorithm reduces to the NCMA. In (11), for $R=1$, the step-size $\mu$ affects the error function as shown in Fig. 2. Note that, $\Psi\left(y_{k}\right)=0$ for $\left|y_{k}\right|=1$ which is the CM property. Unlike the NCMA, when $\mu \in(0,1)$, the ZNL in the SCS algorithm does not perform any hard limiting on the equaliser output. From this view point, the NCMA update is derived in an extreme condition.

From (11), (12), the update equation of the SCS-1 algorithm is obtained as

$$
\theta_{k+1}=\theta_{k}+\frac{\mu}{\left\|X_{k}\right\|_{2}^{2}}\left(1-\frac{\left|y_{k}\right|}{R}\right) y_{k} X_{k}
$$

This corresponds to retaining the first term in the series expansion of (12) around $\left(1-\frac{\left|y_{k}\right|}{R}\right)=0$, and hence the name SCS- 1 .

The value of $R$ in the SCS-1 algorithm can be determined from the gradient vector of the unnormalised algorithm [6], based on the approach in [3] as

$$
R=\frac{E\left\{\left|a_{k}\right|^{3}\right\}}{E\left\{\left|a_{k}\right|^{2}\right\}}
$$

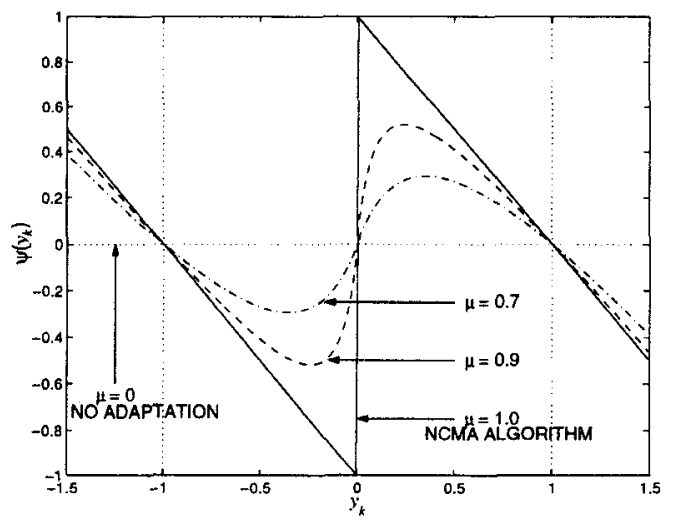

Figure 2: Error function $\Psi\left(y_{k}\right)$ with $R=1$.

\section{STATIONARY POINTS FOR AR CHANNELS}

For an $\operatorname{AR}(p)$ channel of the form [2]

$$
C(z)=\frac{1}{1+\alpha z^{-p}}, \quad 0<|\alpha|<1
$$

we show that Ding-type ULS does not exist for the SCS-1. Let us assume an FIR equaliser

$$
\theta(z)=\sum_{i=0}^{m} \theta_{i} z^{-i}, m \geq p
$$

We start by hypothesising that as in most BE algorithms that use signals sampled at the symbol rate, ULS of the type $\theta_{*}=\left[\begin{array}{llll}0 & 0 & \ldots & \theta_{m}\end{array}\right]^{T}\left(\theta_{m} \neq 0\right)$ exists [2]. To be a stationary point $\theta_{*}$ must satisfy

$$
E\left\{\frac{1}{\left\|X_{k}\right\|_{2}^{2}}\left(1-\frac{\left|y_{k}^{\theta_{*}}\right|}{R}\right) y_{k}^{\theta_{*}} X_{k}\right\}=\mathbf{0}
$$

where $y_{k}^{\theta_{*}}=x_{k-m} \theta_{m}$ and $\mathbf{0}$ is the null vector. In other words

$$
E\left\{\frac{1}{\left\|X_{k}\right\|_{2}^{2}}\left(1-\frac{\left|x_{k-m}\right|\left|\theta_{m}\right|}{R}\right) x_{k-m} x_{k-i}^{*}\right\} \theta_{m}=0
$$

for $i=0,1, \ldots, m$. Due to (15), $x_{k-1}, \ldots, x_{k-p+1}$ are statistically independent for i.i.d channel input [2]. If the normalisation $\left\|X_{k}\right\|_{2}^{2}$ did not exist then using this independence, the system of equations in (18) would reduce to a single equation (for statistically stationary $x_{k}$ ), which yields

$$
\left|\theta_{m}\right|=\frac{E\left\{\left|x_{k}\right|^{2}\right\}}{E\left\{\left|x_{k}\right|^{3}\right\}}
$$

However, in our case, due to the normalisation we have the following $m+1$ equations

$$
\left|\theta_{m}\right|=\frac{E\left\{\left(\left\|X_{k}\right\|_{2}^{2}\right)^{-1} x_{k-m} x_{k-i}^{*}\right\}}{E\left\{\left(\left\|X_{k}\right\|_{2}^{2}\right)^{-1}\left|x_{k-m}\right| x_{k-m} x_{k-i}^{*}\right\}}
$$

for $i=0,1, \ldots, m$. Since, in general there is no unique $\theta_{m}$ that satisfies all equations in (20), the SCS-1 algorithm does not have ULS of the type $\theta_{*}=\left[\begin{array}{llll}0 & 0 & \ldots & \theta_{m}\end{array}\right]^{T}$ for the channel in (15). 


\section{SIMULATIONS}

In order to support the result in the previous section, the channel is chosen as

$$
C_{1}(z)=\frac{1}{1+0.60 z^{-1}}
$$

A two-tap equaliser is used; $\theta=\left[\theta_{0} \theta_{1}\right]^{T}$ and BPSK symbols are transmitted. As clearly indicated by Fig. 3(a), there are no ULS for the SCS-1 algorithm. However, if normalisation of the gradient vector is omitted in (13), two ULS appear around $\theta_{*} \approx \pm\left[\begin{array}{ll}0 & 0.6\end{array}\right]$ as shown in Fig. 3(b).

A maximum-phase channel with the transfer function

$$
C_{2}(z)=0.49+0.70 z^{-1}+z^{-2}
$$

is also used. The EPSs for the SCS-1 and NCMA are shown in Fig. 4. The NCMA clearly has many ULSs but the SCS1 algorithm has only two DLSs. Convergence trajectories from various initial conditions are shown Fig. 5, where the SNR at the channel output is chosen as 20dB. For some initial equaliser parametrisations NCMA converges to ULS whereas the SCS-1 algorithm converges to DLS consistently.

Finally, the channel is chosen to have complex impulse response coefficients;

$$
\begin{aligned}
C_{3}(z)= & 1+(0.50+j 1.11) z^{-1} \\
& +(0.25-j 0.84) z^{-2}-(0.35-j 0.07) z^{-3}
\end{aligned}
$$

and 16-QAM symbols are transmitted. An 11-tap equaliser is employed with its center-tap initialised to $1+j$. The Open-Eye Measure (OEM) is obtained for the NCMA and SCS-1 algorithms which is defined as

$$
\operatorname{OEM}(k)=(M-1) \frac{\left\|T_{k}\right\|_{1}-\left\|T_{k}\right\|_{\infty}}{\left\|T_{k}\right\|_{\infty}}
$$

where $T_{k}$ represents the combined channel and equaliser and $M$ is the number of amplitude levels in $a_{k}$. The eye is open (closed), if $O E M(k)<1(\geq 1)$. The SNR is $30 \mathrm{~dB}$ and $\mu=0.1$ for both algorithms. The results are averaged over 20 trials and shown in Fig. 6. Both algorithms open the eye and the SCS-1 algorithm has better steady-state performance.

\section{CONCLUSIONS}

The family of SCS algorithms is presented for blind channel equalisation. The proposed algorithms have a more favourable, error performance surface than the well-known algorithms in the same class. Currently research in this area is concentrated upon the generalisation of the properties of the error surface, imposing multiple constraints [11] and extension of this algorithm to fractionally-spaced equalisers.

\section{REFERENCES}

[1] A. Benveniste, M. Goursat, and G. Ruget. Robust identification of a nonminimum phase system:Blind adjustment of a linear equaliser in data communications. IEEE Trans. Automat. Contr., AC-25(3):385399, June 1980.

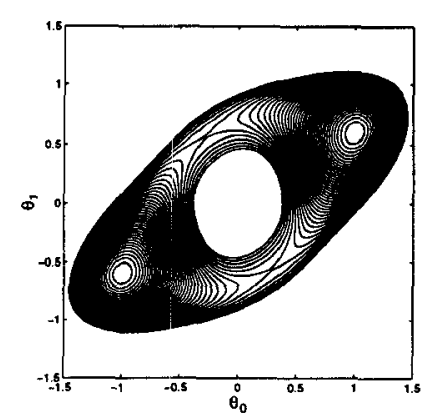

(a)

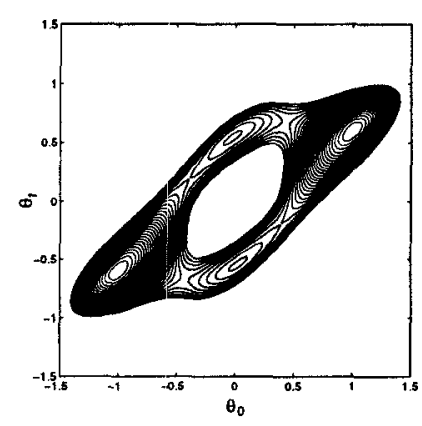

(b)

Figure 3: EPSs for $C_{1}(z)$, (a) with and (b) without the normalisation in the SCS-1 algorithm.

[2] Z. Ding, C. R. Johnson Jr., and R. A. Kennedy. Global convergence issues with linear blind adaptive equalisers. In S. Haykin, editor, Blind Deconvolution, chapter 3, pages 60-120. Englewood Cliffs, NJ: PrenticeHall, 1994.

[3] N. Godard. Self-recovering equalisation and carrier tracking in two-dimensional data communications systems. IEEE Trans. Commun., COM-28(10):18671875, Nov. 1980.

[4] K. Hilal and P. Duhamel. A convergence study of the constant modulus algorithm leading to a NormalisedCMA and Block-Normalised-CMA. In EUSIPCO-92, Brussels, Belgium, pages 135-138, Aug. 1992.

[5] R. W. Lucky. Techniques for adaptive equalisation of digital communication systems. Bell. Syst. Tech, Journal, 45:255-286, 1966.

[6] C. Papadias. Methodés d'égalisation et d'identification aveugle pour les canaux linéaires. $\mathrm{PhD}$ thesis, Ecole Nationale Supérieure des Télécommunications, May 1995.

[7] C. B. Papadias and D. T. M. Slock. New adaptive blind equalisation algorithms for constant modulus constellations. In ICASSP94, volume 3, pages 321-324, May 1994. 


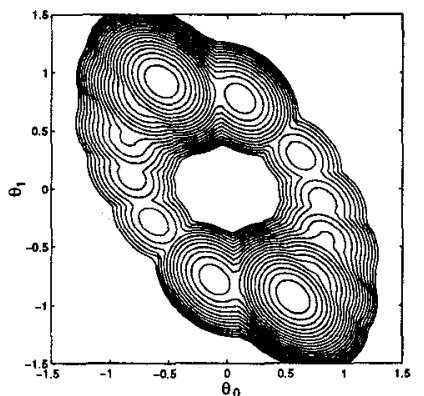

(a)

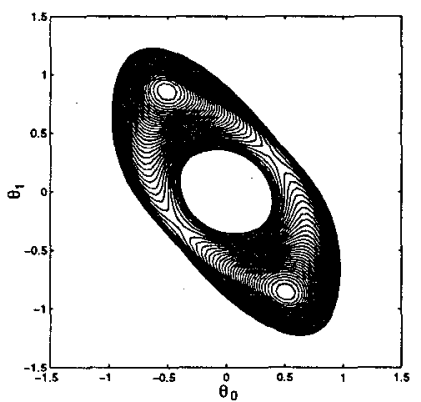

(b)

Figure 4: EPSs of the (a) NCMA and (b) SCS-1 algorithms for $C_{2}(z)$.

[8] G. Picchi and G. Prati. Blind equalisation and carrier recovery using a 'Stop-and-Go' decision-directed algorithm. IEEE Trans. Commun., COM-35:679-682, 1987.

[9] Y. Sato. A method of self-recovering equalisation for multilevel amplitude modulation systems. IEEE Trans. Commun., COM-23(6):679-682, June 1975.

[10] O. Shalvi and E. Wienstein. New criteria for blind deconvolution of nonminimum phase systems (channels). IEEE Trans. Inform. Theory, IT-36(2):312-321, Mar. 1990.

[11] O. Tanrikulu, B. Baykal, A. G. Constantinides, and J. A. Chambers. Soft constraint satisfaction (SCS) blind channel equalisation algorithm. Submitted to Int. Journal of Adaptive Control and Signal Process. ing, 1996. Special Issue on Adaptive Channel Equalisation.

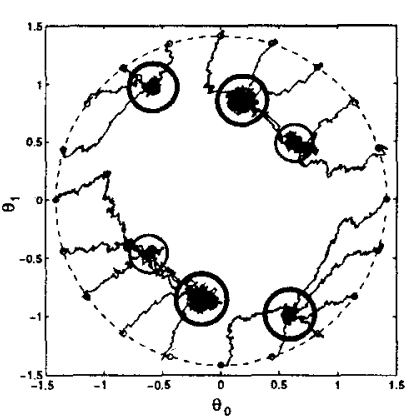

(a)

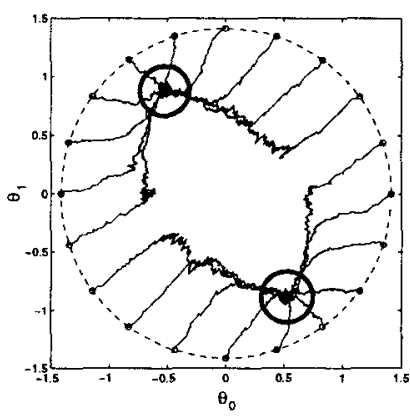

(b)

Figure 5: Convergence trajectories of the (a) NCMA and (b) SCS-1 algorithms for $C_{2}(z)$.

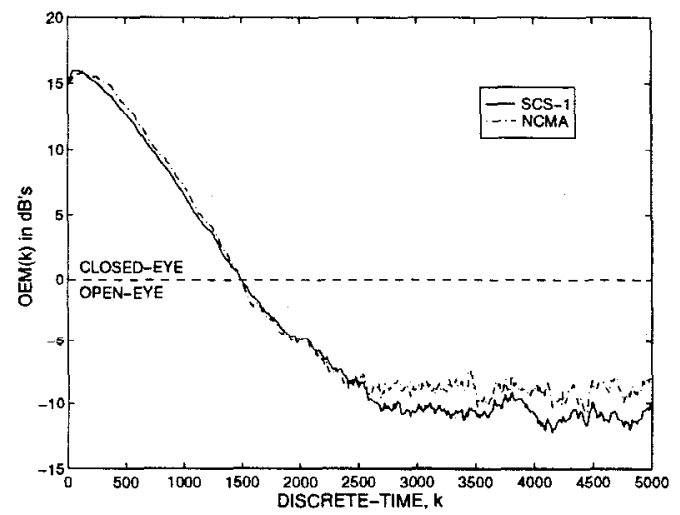

Figure 6: Open-eye Measure for $C_{3}(z)$ and 16-QAM signal with NCMA and SCS-1 algorithms. 